\title{
Phosphates naturels et alimentation du bétail en zone sahélienne I. Influence sur la santé et la croissance du zébu G obra
}

\author{
S.T. Fall ${ }^{1}$ G. Sawadogo ${ }^{2}$ M. Diop ${ }^{1}$
}

Mots-clés

Bovin - Zébu Gobra - Phosphate naturel - Complémentation - Pâturage Sahel - Sénégal.

\begin{abstract}
Résumé
Pour préciser le mode d'utilisation des phosphates naturels en alimentation animale, deux essais ont été menés au Sénégal. Des zébus Gobra ont été supplémentés avec le phosphate de Taiba (TAP 3 à 4 p. 100 matière sèche de fluor) et celui de Thiès (THP 0,8 à 1 p. 100 MS de fluor), à Dahra et en milieu contrôlé à Sangalcam, au Sénégal en zone sahélienne. Dans I'essai 1, la supplémentation des zébus $\mathrm{G}$ obra a été effectuée sur pâturage naturel au $\mathrm{Crz}$ de Dahra pendant trois ans. Les doses appliquées étaient de $50 \mathrm{~g}$ de phosphate de Taiba, selon les modes continu et discontinu, respectivement pour les lots 1 et 2 (pour ce dernier lot, la distribution selon le mode discontinu a été abandonnée dans le courant de la deuxième année par suite d'une faible ingestion de phosphate). Elles étaient de 50 et de $100 \mathrm{~g}$ de phosphate de Thiès respectivement pour les lots 3 et 4 et de $65 \mathrm{~g}$ de poudre d'os pour le lot 5 , selon un mode continu pour l'ensemble des lots. Le lot 6 témoin n'a reçu aucun supplément. Les animaux ont été supplémentés en saison sèche pendant huit à neuf mois par an (entre octobre et juin). Dans l'essai 2, quatre lots ont été supplémentés en milieu contrôlé pendant neuf mois. Les bovins ont reçu $50 \mathrm{~g}$ de TAP en mode continu, $50 \mathrm{~g}$ de TAP en mode discontinu et $200 \mathrm{~g}$ de THP en mode continu respectivement pour les lots 1,2 et 3 . Ces animaux ont été comparés au lot 4 témoin non supplémenté. Pour les deux essais, les mesures concernaient le contrôle quotidien de la consommation des phosphates, le suivi pondéral par une double pesée mensuelle des lots et des examens cliniques portant sur l'état général, l'appareil locomoteur et les dents pour détecter les signes de fluorose. Un contrôle biochimique a porté sur l'analyse de la phosphatémie et de la fluorurie. Les résultats ont mis en évidence une bonne tolérance des doses appliquées. L'influence de la supplémentation minérale sur la croissance des animaux n'a cependant pas été significative $(P<0,05)$. L'innocuité du produit permet de suggérer un test de longue durée en milieu éleveur, plus propice à une démonstration de l'effet bénéfique de la supplémentation minérale sur les performances zootechniques du zébu Gobra.
\end{abstract}

\section{INTRODUCTION}

Les carences minérales figurent parmi les principales causes de maladies et de baisses de production en zone sahélienne. L'aphosphorose en particulier est l'étiologie primitive de maladies infectieuses comme le botulisme (3), de fractures, et de troubles de la consommation et de la reproduction, très répandus dans ces régions.

1. Institut sénégalais des recherches agricoles, Laboratoire national d'élevage et de recherches vétérinaires, BP 2057, Dakar, Sénégal

2. Ecole inter-Etats des sciences et médecines vétérinaires, Service de chimie et biophysique médicales, BP 5077, Dakar, Sénégal
Les déséquilibres en minéraux sont rapportés dans beaucoup de pays tropicaux (7). Au Sénégal, des recherches ont été menées dans ce domaine depuis les années 60 , dans la principale zone d'élevage, le Ferlo. Une synthèse d'études cliniques et biochimiques a permis de mettre en évidence des carences en phosphore (3), sodium, cuivre et zinc (14).

La complémentation minérale en général et phosphocalcique en particulier est donc une nécessité pour améliorer la santé et la productivité des troupeaux $(8,11,12)$. Cependant, les compléments minéraux sont chers et peu accessibles dans les zones d'élevage.

Les phosphates naturels sont une source potentielle de calcium et de phosphore. Ils sont disponibles dans la plupart des pays du Sahel dans des mines peu profondes. Leur utilisation en alimentation du 
bétail peut être limitée par un rapport phospho/calcique non optimal, une digestibilité médiocre $(4,15,17)$ et surtout une teneur en fluor élevée qui risque de provoquer la fluorose chez les animaux qui les consomment $(6,7,20,29)$.

Les premiers essais d'utilisation des phosphates naturels datent du début du siècle aux Etats-Unis. Les résultats rapportés ont souvent été contradictoires. En Afrique, les phosphates ont été déclarés toxiques et dangereux (5) (Velu, 1933, laboratoire du Service d'élevage du Maroc). Au contraire, Calvet et coll. (2), Lerman et coll. (communic.) et la Société des minerais de Thiès (Smt) (28) ont rapporté une parfaite tolérance du produit. Serres et Bertaudières ont mis en évidence l'influence du type de phosphate et du mode de distribution sur leur tolérance par les bovins au Tchad (26).

L'efficacité zootechnique des phosphates naturels fait également l'objet de controverses. Pour Calvet et coll. (2) le phosphate ferroalumino-calcique est moins efficace que le phosphate bicalcique, alors que Lerman (communic.) et la Smt (28) rapportent une équivalence entre les deux compléments minéraux. Le phosphate ferroalumino-calcique ou polyphos est commercialisé aujourd'hui en Afrique et en Europe.

Pour définir les méthodes d'utilisation des phosphates naturels comme supplément minéral pour les ruminants et examiner leur efficacité zootechnique sur le zébu Gobra, des expériences de supplémentation du zébu Gobra ont été menées au Crz de Dahra (nord-est du Sénégal) et à Sangalcam (région du Cap-Vert, Sénégal) de 1987 à 1990. L'objectif a été d'étudier les caractéristiques biochimiques de minerais de phosphates naturels disponibles au Sénégal en vue de définir des méthodes optimales de leur utilisation dans la prévention des carences en phosphore. Pour cela, les éléments suivants ont été étudiés :

- la dose quotidienne optimale de phosphates naturels de Taiba et de Thiès et la durée limite de complémentation pour des zébus Gobra ;

- le mode et la périodicité de distribution les plus appropriés ;

- l'influence de ces phosphates naturels sur l'évolution pondérale de taurillons Gobra et l'évaluation des risques de toxicité du fluor.

\section{MATERIEL ET METHODES}

\section{Le site expérimental}

Les expériences se sont déroulées en milieu naturel au Centre de recherches zootechniques de Dahra situé dans le sud-ouest du sahélienne typique avec une longue saison sèche de 9 à 10 mois (entre octobre et juin) et une courte saison humide de 2 à 3 mois et demi (entre juillet et octobre). La pluviométrie a varié de 470 (en 1987) à 225 mm en 1990. Les sols du Ferlo sont pauvres en éléments nutritifs et en minéraux en particulier (3). Du point de vue ressources en eau, il y a des mares temporaires à durée de vie très courte pendant la période hivernale et post-hivernale, des puits traditionnels peu profonds et surtout des forages profonds qui abreuvent les troupeaux pendant la majeure partie de l'année. Leurs eaux sont également pauvres en minéraux (13). Lors de la deuxième année d'expérience, pour mieux surveiller la consommation de phosphates, des lots ont été mis en stabulation à Sangalcam, une station de l'Isra située dans la banlieue de Dakar.

\section{Le plan expérimental}

\section{Les animaux et leur alimentation}

- Essai 1

En milieu extensif, 78 taurillons de race Gobra âgés d'un à deux ans et d'un poids moyen de $140 \mathrm{~kg}$ ont été déparasités, identifiés et vaccinés contre la peste bovine, la péripneumonie contagieuse bovine et le botulisme, puis divisés en six lots de 13 chacun. Leur alimentation était uniquement basée sur le pâturage naturel. Ils ont été maintenus dans la parcelle A de juin 1987 à janvier 1989. D'une superficie de 429 hectares, la biomasse disponible en novembre 1987 a été de $1270 \mathrm{~kg} / \mathrm{ha}$. Pour augmenter la charge et exacerber le besoin en phosphore, le troupeau a été transféré sur les parcelles 8 et 9 (130 ha) à partir de janvier 1989. L'essai a duré trois ans (de juin 1987 à juin 1990). Les animaux ont été abreuvés deux fois par jour en moyenne. Le complément minéral a été distribué pendant la saison sèche (d'octobre à juin), le matin à jeun entre 9 et $12 \mathrm{~h}$ selon le plan expérimental décrit au tableau I. Une distribution directe, sans contention a été faite, dans des récipients individuels, après attache au piquet. Pour augmenter l'appétibilité des phosphates, différents condiments lui ont été associés à raison de $20 \mathrm{~g}$ mélangés à la dose quotidienne. La mélasse, le sel (chlorure de sodium), le son de blé et la graine de coton ont été comparés. L'utilisation de ces supports pendant une semaine chacun a permis de sélectionner la mélasse comme le condiment le mieux accepté par les animaux. Les apports de minéraux sont décrits au tableau II.

- Essai 2

En milieu contrôlé, 40 veaux sevrés et 40 taureaux mâles adultes de race Gobra, ayant subi les traitements préliminaires (déparasitage et vaccinations), ont été divisés en quatre lots mis en stabulation à Sangalcam. Ils ont reçu de la fane d'arachide de juillet à

Tableau I

Distribution de minéraux en milieu extensif ( $D$ ahra)

$\begin{array}{lccccc}\text { Lots } & \text { I } & \text { II } & \text { III } & \text { IV } & \begin{array}{c}\text { V } \\ \text { VI } \\ \text { (témoin) }\end{array} \\ \begin{array}{lcccc}\text { Complément } \\ \text { Minéral }\end{array} & \begin{array}{c}\text { Phosphate } \\ \text { de Taiba }\end{array} & \begin{array}{c}\text { Phosphate } \\ \text { de Taiba }\end{array} & \begin{array}{c}\text { Phosphate } \\ \text { de Thiès }\end{array} & \begin{array}{c}\text { Phosphate } \\ \text { de Thiès }\end{array} & \begin{array}{c}\text { Poudre } \\ \text { d'os }\end{array} \\ \begin{array}{l}\text { Dose } \\ \text { Quotidienne }\end{array} & 50 \mathrm{~g} & 50 \mathrm{~g} & 50 \mathrm{~g} & 100 \mathrm{~g} & 65 \mathrm{~g} \\ \begin{array}{l}\text { Mode de } \\ \text { Distribution }\end{array} & \text { Continu } & \begin{array}{c}\text { Discontinu } * \\ \text { (1 mois sur 2) }\end{array} & \text { Continu } & \text { Continu } & \text { Continu } \\ \mathrm{N} & 13 & 13 & 13 & 13 & 13\end{array}$

* La distribution discontinue a été interrompue au bout d'un an à cause de la faiblesse de la consommation de phosphate observée au sein de ce lot 
octobre 1989, puis un aliment composé à base de coque d'arachide, de mélasse, d'urée, de tourteau d'arachide et de graines de coton, d'octobre 1989 à juillet 1990 (tableau III).

La dose quotidienne de phosphate a été mélangée à l'aliment complet selon le plan décrit au tableau IV. Les apports complémentaires en minéraux sont présentés au tableau V.

\section{Les mesures}

- La consommation des minéraux

En milieu extensif, le numéro des animaux qui ont volontairement consommé le complément a été noté tous les jours. En milieu contrôlé la totalité des phosphates a toujours été consommée.

- Le prélèvement du fourrage consommé sur pâturage

Un échantillonnage représentatif du fourrage ingéré a été mensuellement effectué par la technique de la « collecte du berger » (18) pour analyse chimique en laboratoire.

\section{- L'examen clinique des troupeaux}

Pour détecter précocement les signes éventuels d'intoxication au fluor, l'examen clinique mensuel du troupeau a porté sur l'état général, l'appareil osseux (par palpation et pression des maxillaires, des côtes et des métatarsiens pour détecter des excroissances osseuses) et l'appareil bucco-dentaire à la recherche d'une coloration brune noirâtre, d'une rugosité ou d'une érosion éventuelle des dents.

- L'examen post mortem de l'appareil osseux

Pour détecter les signes de fluorose, l'appareil osseux a été examiné après abattage de deux animaux par lot en fin d'expérience. Ces examens ont porté sur les maxillaires inférieurs, les métatarsiens et les côtes pour rechercher les signes osseux de fluorose tels que les excroissances et les rugosités osseuses.

- Croissance des animaux

Pour suivre l'évolution pondérale des animaux, une triple pesée de démarrage, puis une double pesée mensuelle et une triple pesée finale ont été effectuées.

\section{Analyses chimiques}

Des prélèvements mensuels de sang et d'urines ont été effectués. Les analyses chimiques ont concerné le fourrage ou l'aliment composé distribué en milieu contrôlé, les compléments minéraux (phosphates et poudre d'os), le plasma sanguin, les urines et les os après abattage des animaux. Le calcium et le phosphore ont été déterminés par spectrophotométrie d'absorption atomique. Le fluor a été analysé par polarographie avec utilisation d'une électrode spécifique (19). Sur les échantillons de fourrages les dosages ont porté sur la matière sèche, les cendres, les matières azotées totales, le calcium et le phosphore (1). Sur les différents types de phosphates la solubilité à l'acide citrique 2 p. 100 (10) et les teneurs en phosphore (1) et fluor par polarographie ont été déterminées (19).

\section{Analyses statistiques}

La signification des différences entre lots a été étudiée par l'analyse de variance. L'influence du lot, de l'animal, du mois, de l'année et de leur interaction a été appréciée par le test de Duncan (25).

\section{RESU LTATS}

\section{Caractéristiques chimiques et ingestibilité des compléments minéraux}

Les compositions minérales des phosphates et de la poudre d'os sont rapportées dans le tableau VI. Les phosphates de Taiba et de
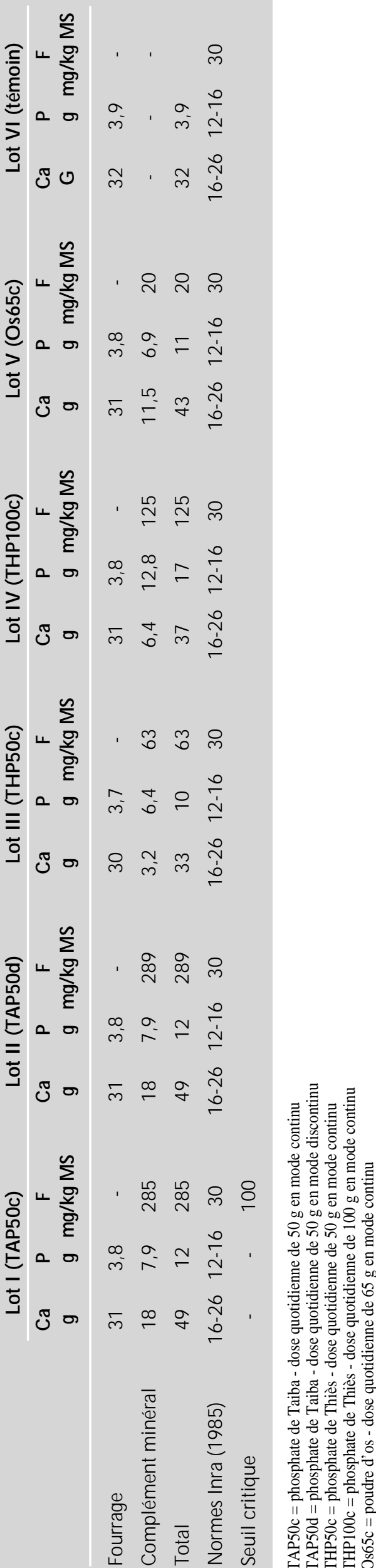


\section{Tableau III}

Alimentation des bovins en milieu contrôlé à Sangalcam

\begin{tabular}{|c|c|c|c|c|c|c|c|}
\hline \multirow[t]{2}{*}{ Période } & \multirow[t]{2}{*}{ Animaux } & \multirow{2}{*}{$\begin{array}{l}\text { Composition } \\
\text { de la ration }\end{array}$} & \multirow{2}{*}{$\begin{array}{l}\text { D istribué } \\
\text { kg/tête/j }\end{array}$} & \multicolumn{4}{|c|}{ Apport total } \\
\hline & & & & UFV & MAD & $\mathrm{Ca}$ & $\mathbf{P}$ \\
\hline \multirow{4}{*}{$\begin{array}{l}\text { D'août à } \\
\text { déc. } 1989\end{array}$} & Jeunes & Fourrage* & 4 & 2,7 & 340 & 57 & 12 \\
\hline & & Complément ** & 1 & & & & \\
\hline & Taureaux & Fourrage & 7 & 4 & 520 & 99 & 16 \\
\hline & & Complément & & 1 & & & \\
\hline \multirow{2}{*}{$\begin{array}{l}\text { De déc. } 1989 \\
\text { à mai } 1990\end{array}$} & Jeunes & Aliment complet $1^{* * *}$ & 5 & 2,5 & 355 & 7 & 6 \\
\hline & Taureaux & Aliment complet 1 & 7 & 3,5 & 497 & 9,8 & 8,4 \\
\hline \multirow{2}{*}{$\begin{array}{l}\text { De mai à } \\
\text { juil. } 1989\end{array}$} & Jeunes & Aliment complet $2^{* * * *}$ & 5 & 2,5 & 200 & 19 & 6,5 \\
\hline & Taureau & Aliment complet 2 & 8 & 4 & 320 & 27 & 11 \\
\hline
\end{tabular}

* Fourrage : fane d'arachide

** Complément : graine de coton mélassée à 25 p. 100

*** Composition de l'aliment complet 1 (pourcentages) : coque d'arachide $(52,8)$; mélasse $(27)$; graine de coton (14); urée (1); tourteau d'arachide (5); sel $(0,2)$

**** Composition de l'aliment complet 2 (pourcentages) : coque d'arachide $(54,6)$; mélasse $(20)$; maïs broyé $(15)$; graine de coton $(10)$; urée $(0,25)$; sel $(0,15)$

Tableau IV

Distribution du complément minéral à Sangalcam

\begin{tabular}{lcccc} 
Lots & I & II & III & IV* \\
\hline $\begin{array}{l}\text { Complément } \\
\text { Minéral }\end{array}$ & $\begin{array}{c}\text { Phosphate } \\
\text { de Taiba }\end{array}$ & $\begin{array}{c}\text { Phosphate } \\
\text { de Taiba }\end{array}$ & $\begin{array}{c}\text { Phosphate } \\
\text { de Thiès }\end{array}$ & - \\
$\begin{array}{l}\text { Dose } \\
\text { Q uotidienne }\end{array}$ & $50 \mathrm{~g}$ & $50 \mathrm{~g}$ & $200 \mathrm{~g}$ & - \\
$\begin{array}{l}\text { Mode de } \\
\text { Distribution }\end{array}$ & Continu & $\begin{array}{c}\text { Discontinu } \\
\text { (1 mois sur 2) }\end{array}$ & Continu & - \\
N jeunes & 10 & 10 & 10 & 10 \\
\multicolumn{1}{c}{ Adultes } & 10 & 10 & 10 & 10
\end{tabular}

* Lot témoin

\section{Tableau V}

Caractéristiques chimiques des compléments minéraux

\section{Minéraux}

\section{Calcium (\% MS)}

Phosphore (\% MS)

Fluor (\% MS)

Magnésium (\% MS)

Aluminium (\% MS)

Silice (\% MS)

Fer $(\% \mathrm{MS})$

$M$ anganèse (\% MS)

\section{Rapport Ca/P}

Solubilité

à l'acide citrique $2 \%$

Disponibilité biologique*

Digestibilité**

\section{Intermédiaire Intermédiaire}

\section{Phosphate de Taiba}

36

15,8

12,8

3,7

0,01

0,56

2,66

0,37

0,030

2,2

45

ND

20

* D'après Conrad et coll., 1985

** D'après Guéguen, 1994
Tableau V

Apports de compléments minéraux à Sangalcam

\begin{tabular}{lccc} 
Lots & $\begin{array}{c}\text { Calcium } \\
\text { (g/tête/jour) }\end{array}$ & $\begin{array}{c}\text { Phosphore } \\
\text { (g/tête/jour) }\end{array}$ & $\begin{array}{c}\text { Fluor } \\
\text { (mg/kg MS) }\end{array}$ \\
\hline I (TAP50C) & 18 & 8 & 285 \\
II (TAP50d) & 18 & 8 & 285 \\
III (THP200C) & 13 & 26 & 250 \\
IV (témoin) & 0 & 0 & 0
\end{tabular}

TAP50 $\mathrm{c}=$ phosphate de Taiba - dose quotidienne de $50 \mathrm{~g}$ en mode continu TAP50d = phosphate de Taiba - dose quotidienne de $50 \mathrm{~g}$ en mode discontinu THP200c $=$ phosphate de Thiès - dose quotidienne de $200 \mathrm{~g}$ en mode continu
Thiès avaient des teneurs en phosphore voisines (16 et 13 p. 100 respectivement). Le premier était cependant six fois plus riche en calcium que le second qui avait un rapport phospho/calcique peu favorable à son absorption.

La solubilité citrique du phosphate de Taiba était supérieure à celle du phosphate de Thiès (45 contre $32 \mathrm{p}$. 100) dont l'utilisation digestive était médiocre (digestibilité réelle $=20$ p. 100) (16). La teneur en fluor du phosphate de Thiès était cependant moins élevée que celle du phosphate de Taiba ( 0,8 contre 3,7 p. 100). Les risques de toxicité au fluor étaient encore amoindris par une forte teneur en alumine, élément qui joue un rôle inhibiteur dans l'absorption du fluor.

La poudre d'os (tableau VI) avait une teneur en phosphore (11 p. 100) plus faible que celle des phosphates. Son rapport phospho/calcique était optimal et sa disponibilité biologique élevée (7). C'est un complément minéral de choix dont la comparaison avec les phosphates a pour but d'apprécier leur qualité.

L'ingestion volontaire des compléments minéraux à Dahra a été caractérisée par d'importantes variations (tableau VII), liées au type de complément minéral, à la saison, à l'année, à l'individu et au support (figure 1).

La poudre d'os a été mieux appétée que les phosphates et le phosphate de Thiès mieux accepté que celui de Taiba. 


\section{Tableau VII}

Evolution de la consommation du complément minéral offert à Dahra : valeurs moyennes mensuelles (nombre d'animaux ayant consommé le complément partiellement ou en totalité exprimé en pourcentage du nombre d'animaux par lot (écart type))

\begin{tabular}{|c|c|c|c|c|c|}
\hline & Lot I (TAP50C) & Lot II (TAP50d) & Lot III (THP50C) & Lot IV (THP100C) & Lot V (0 s65c) \\
\hline \multicolumn{6}{|l|}{ Année 1987} \\
\hline Juin & $80(16)$ & $72(22)$ & $81(15)$ & 71 (17) & $71(21)$ \\
\hline Juillet & $96(6)$ & - & $99(2)$ & $95(6)$ & $90(5)$ \\
\hline Novembre & $54(17)$ & $51(17)$ & $84(10)$ & $86(11)$ & $92(8)$ \\
\hline Décembre & $49(18)$ & $43(12)$ & $86(6)$ & $94(8)$ & $93(2)$ \\
\hline \multicolumn{6}{|l|}{ Année 1988} \\
\hline Janvier & $45(11)$ & - & $86(2)$ & $87(7)$ & $92(0)$ \\
\hline Février & $45(12)$ & $40(10)$ & $89(4)$ & $95(5)$ & $93(3)$ \\
\hline Mars & $58(11)$ & - & $83(7)$ & $87(12)$ & $91(4)$ \\
\hline Avril & $52(11)$ & 41 (16) & $84(6)$ & $76(10)$ & $96(6)$ \\
\hline Mai & $65(7)$ & - & $90(3)$ & $91(6)$ & $95(6)$ \\
\hline Juin & $68(6)$ & $49(8)$ & $90(6)$ & $96(9)$ & $91(5)$ \\
\hline Juillet & $81(14)$ & - & $95(4)$ & $97(5)$ & $89(4)$ \\
\hline Août & $65(31)$ & $74(26)$ & $86(26)$ & $75(33)$ & $87(25)$ \\
\hline Décembre & $3(6)$ & $4(8)$ & $23(11)$ & $7(8)$ & $58(21)$ \\
\hline \multicolumn{6}{|l|}{ Année 1989} \\
\hline Janvier & $1(4)$ & $3(6)$ & $21(13)$ & $5(4)$ & $49(20)$ \\
\hline Février & $19(7)$ & $14(8)$ & $27(7)$ & $16(6)$ & $51(16)$ \\
\hline Mars & $26(8)$ & $21(4)$ & $38(12)$ & $25(12)$ & $89(10)$ \\
\hline Avril & 31 (14) & $18(9)$ & $68(14)$ & 49 (15) & $93(6)$ \\
\hline Mai & $31(6)$ & $18(5)$ & $59(17)$ & $52(18$ & $80(18)$ \\
\hline Juin & 39 (17) & $41(20)$ & $57(24)$ & $47(24$ & 85 (14) \\
\hline Novembre & $22(17)$ & $20(15)$ & $40(12)$ & $26(6)$ & $63(17$ \\
\hline Décembre & $12(9)$ & - & $33(9)$ & $21(6)$ & 51 (13) \\
\hline \multicolumn{6}{|l|}{ Année 1990} \\
\hline Janvier & $23(6)$ & $22(18)$ & 46 (19) & 35 (17) & 71 (19) \\
\hline Février & $42(11)$ & $40(10)$ & $70(12)$ & $62(11)$ & 69 (15) \\
\hline Mars & $44(8)$ & $47(9)$ & $74(10)$ & $66(9)$ & 74 (9) \\
\hline Avril & $32(9)$ & $33(10)$ & $52(15)$ & 48 (13) & $59(16)$ \\
\hline Mai & 31 (11) & $20(13)$ & 71 (11) & $58(14)$ & $72(12)$ \\
\hline Juin & $24(9)$ & $13(11)$ & $62(12)$ & $44(7)$ & 75 (11) \\
\hline
\end{tabular}

TAP50c $=$ phosphate de Taiba - dose quotidienne de $50 \mathrm{~g}$ en mode continu TAP50d = phosphate de Taiba - dose quotidienne de $50 \mathrm{~g}$ en mode discontinu THP50c $=$ phosphate de Thiès - dose quotidienne de $50 \mathrm{~g}$ en mode continu THP100c $=$ phosphate de Thiès - dose quotidienne de $100 \mathrm{~g}$ en mode continu Os $65 \mathrm{c}=$ poudre d'os - dose quotidienne de $65 \mathrm{~g}$ en mode continu

Dès la tombée des premières pluies (juillet - août), les animaux ont commencé à refuser le complément. Ils ne l'ont pas consommé pendant toute la durée de la saison humide et en posthivernage. En revanche, en milieu et fin de saison sèche le taux de consommation des compléments minéraux était plus important, ce qui correspondait sans doute à un besoin en phosphore plus élevé.

L’ingestion volontaire de compléments minéraux a été supérieure en deuxième année. Cela est explicable par un état de carence plus prononcé car la teneur en phosphore du fourrage a été particulièrement faible à cette période $(<2 \mathrm{~g} / \mathrm{kg} \mathrm{MS}$; tableau VIII).

Les écarts types des taux de consommation ont souvent été élevés, traduisant des différences dans le comportement des individus dont la consommation a varié d'un jour à l'autre. Les vents de sable en particulier ont joué un rôle négatif sur l'ingestion volontaire du complément minéral par les animaux.

\section{Le régime alimentaire des animaux}

La dégradation du fourrage de la saison humide à la saison sèche a été mise en évidence. Les apports naturels de phosphore en particulier (moins de $1 \mathrm{~g} / \mathrm{kg} \mathrm{MS}$ ) ont été très insuffisants en 1988 et 1989. Les animaux ont ainsi montré une forte propension à consommer les compléments minéraux en ces périodes.

La teneur du fourrage en fluor a été élevée. En effet, sur 10 analyses la moyenne a été de $1349 \mathrm{mg} / \mathrm{kg}$ MS. Ce chiffre, beaucoup plus élevé que le seuil critique de $100 \mathrm{mg} / \mathrm{kg}$ MS (29), traduit une forte contamination des fourrages par la présence de formations argilophosphatées dans la zone de Linguère (21). Cela est confirmé par les fortes teneurs en insoluble chlorhydrique observées.

A Dahra, les apports de phosphore des lots supplémentés (10 à $12 \mathrm{~g}$ par tête par jour) ont été corrects. Le témoin a eu un déficit très important. Un excès de calcium, aggravé par la présence d'eaux calciques (3), défavorise l'absorption du phosphore. 

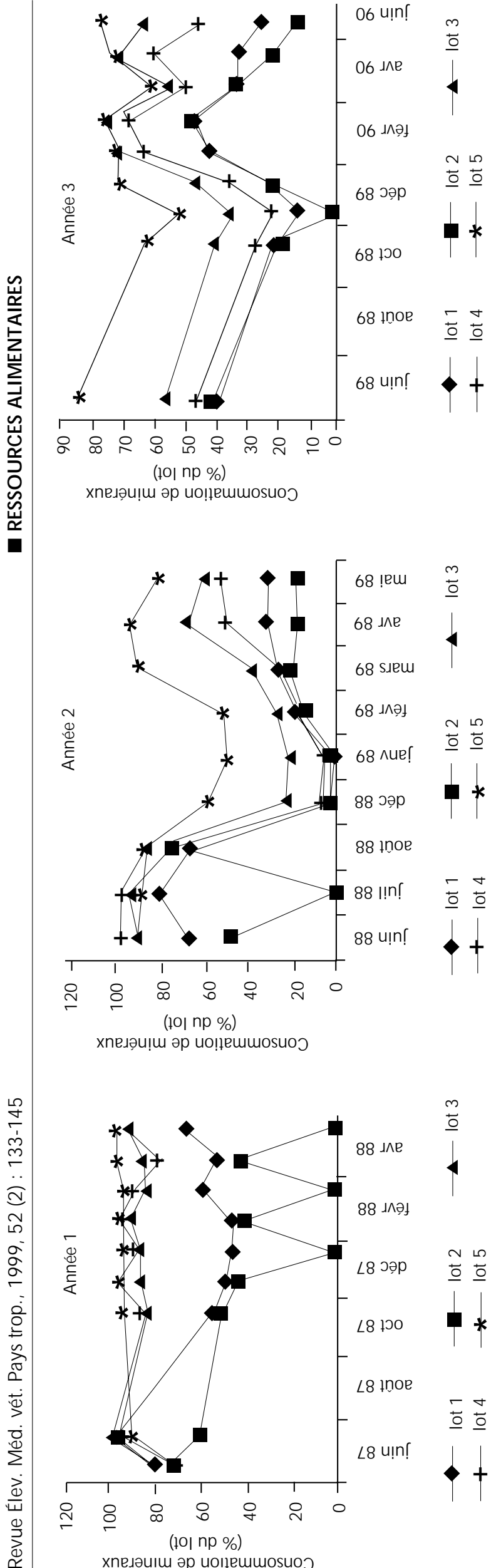

\section{Tableau VIII}

Composition chimique du fourrage ingéré sur pâturage naturel (g/kg MS)

\begin{tabular}{lcccccc} 
Saison* Année & Mo & MAT & $\begin{array}{c}\text { Insoluble } \\
\text { chlorhydrique }\end{array}$ & Ca & P \\
& & \multicolumn{5}{c}{} \\
SSF & 1987 & 933 & 102 & 17 & 5,4 & 1,4 \\
SSC & & 893 & 103 & 66 & 7,5 & 1,2 \\
SH & & 892 & 179 & 45 & 7,4 & 1,8 \\
SSF & 1988 & 940 & 77 & 20 & 5,2 & 0,5 \\
SSC & & - & 54 & 7 & 5,7 & 0,4 \\
SH & & 977 & - & - & - & - \\
SSF & 1989 & 924 & 68 & 39 & 5,2 & 0,4 \\
SSC & & 821 & 66 & 38 & 6,5 & 0,5 \\
SH & & 932 & 132 & 42 & 6,4 & 1 \\
SSF & 1990 & 926 & 63 & 45 & 5,3 & 0,5 \\
SSC & & 916 & 39 & 52 & 5 & 0,3
\end{tabular}

MO : matière organique ; MAT : matières azotées totales

* SSF : saison sèche froide (novembre-février) ; SSC : saison sèche chaude (mars-juin) ; SH : saison humide (juillet-octobre)

Les quantités de fluor ingérées par les lots 1 et 2 (285 mg/ $/ \mathrm{kg} \mathrm{MS})$ ont été près de trois fois supérieures au seuil critique de $100 \mathrm{mg} / \mathrm{kg}$ MS (7, 6). La consommation de ces phosphates pendant quelques années devrait ainsi entraîner la fluorose (29). En revanche, les quantités de fluor absorbées par les lots 3 et 4 (30 mg/kg MS) n'ont pas été très élevées.

En milieu contrôlé, il y a eu un déséquilibre phosphocalcique volontairement recherché. Le régime alimentaire a apporté 6 et $10 \mathrm{~g}$ de phosphore respectivement aux jeunes et aux taureaux. Un déficit de 50 p. 100 des besoins a été partiellement comblé par les apports complémentaires pour les lots 1 et 2 , et totalement pour le lot 3 . En ce qui concerne le fluor, les quantités absorbées (290 mg/kg MS) ont été de beaucoup supérieures au maximum tolérable de $100 \mathrm{mg} / \mathrm{kg}$ MS indiqué par la bibliographie. Les trois lots ont eu des consommations de fluor voisines.

\section{L'examen clinique des animaux}

L'état général des troupeaux a été bon dans l'ensemble. Aucun signe général d'intoxication au fluor n'a été noté. L'appareil osseux n'a pas été atteint. Aucune boiterie ou exostose n'a été observée ni à Dahra ni à Sangalcam. De même, l'appareil bucco-dentaire n'a pas été significativement affecté. Un brunissement des dents accompagné d'une rugosité a été noté dès l'apparition des dents adultes. Ces lésions ont été observées chez des animaux de tous les lots, y compris le lot témoin et celui recevant de la poudre d'os.

\section{La minéralisation osseuse et son imprégnation par le fluor}

Aucune différence significative entre lots n'a été observée en ce qui concerne les matières minérales totales. Une concentration en calcium plus élevée a été observée chez les animaux âgés. L'influence de la supplémentation sur la teneur en phosphore des os n'a pas été significative $(\mathrm{P}<0,05)$ (tableau IX).

Sur 14 analyses, la teneur des os en fluor a varié de 500 à 7060 $\mathrm{mg} / \mathrm{kg}$ MS avec une moyenne $1387 \mathrm{mg} / \mathrm{kg}$ MS ( \pm 1 673). Les os des taureaux ont eu une concentration en fluor plus élevée que ceux des veaux. Parmi les taureaux, les valeurs les plus fortes ont été notées chez les animaux du lot 1 recevant le phosphate de 
Taiba de manière continue ; la valeur de l'un d'entre eux a dépassé largement $(7060 \mathrm{mg} / \mathrm{kg}$ MS) les valeurs usuelles $(5500 \mathrm{mg} / \mathrm{kg}$ MS) (7). Les 13 autres prélèvements ont eu des teneurs en fluor très inférieures au seuil critique, ce qui témoigne d'une mauvaise fixation du fluor sur l'appareil osseux.

\section{Phosphatémie et fluorurie}

En milieu extensif, la phosphatémie des lots traités n'a pas différé significativement $(\mathrm{P}<0,05)$ de celle des lots témoins, à l'exception du lot 5 (poudre d'os) qui, à Dahra au cours de la deuxième année, a présenté une valeur significativement plus élevée $(\mathrm{P}<0,05)$ (figure 2). En revanche, le dosage du fluor dans les urines a montré que l'administration de phosphate s'est accompagnée d'une augmentation de la concentration en fluor des urines (figure 3) avec des valeurs dépassant parfois la limite supérieure tolérée, ce qui peut annoncer l'apparition de signes cliniques de fluorose $(20,27)$

En avril 1990, à Dahra, la fluorurie des animaux des différents lots supplémentés a été très proche. Les possibilités d'excrétion urinaire du fluor semblent avoir été saturées à ce moment-là (figure 3).

En milieu contrôlé, l'examen des résultats biochimiques notamment en ce qui concerne la phosphatémie (figures 4 et 5) n'a pas montré de variations significatives pouvant être rattachées à la complémentation en phosphates naturels, chez les taurillons comme chez les adultes. Le dosage du fluor dans les urines indique cependant qu'il y a eu une différence significative $(\mathrm{P}<0,05)$ entre les lots recevant les phosphates naturels en mode continu et discontinu et les lots témoins (figures 6 et 7). La différence de la concentration urinaire en fluor observée entre les mois d'avril et de mai 1990 traduisait une imprégnation progressive de l'organisme en fluor.

L'excrétion urinaire de fluor est dépendante du type de phosphate (notamment sa concentration en fluor et la solubilité de cet élément), de la dose appliquée, de la durée et du mode de supplémentation (continu ou discontinu). Ainsi, les lots recevant les phosphates de manière continue ont eu une fluorurie plus importante (figure 8).

L'excrétion urinaire de fluor a été plus forte chez les lots recevan le phosphate de Taiba que chez ceux recevant le phosphate de Thiès. La même observation a été faite chez les animaux âgés comparés aux veaux (16 contre 19 à $20 \mathrm{mg} / \mathrm{kg} \mathrm{MS}$ ).

Il y a donc eu un déstockage important du fluor par voie urinaire. L'âge des bovins, le mode et la durée de distribution ainsi que le type de phosphate ont eu une grande influence sur l'élimination du fluor.

Enfin, les témoins ont eu une fluorurie de 0,5 à $2 \mathrm{mg} / \mathrm{kg} \mathrm{MS}$. Ce niveau semble être compatible avec un métabolisme normal de l'animal.

\section{Tableau IX}

Teneur en minéraux des os des animaux à Sangalcam

\begin{tabular}{|c|c|c|c|c|c|}
\hline Lots & Groupe & $\begin{array}{c}\text { Cendres } \\
\text { (g/kg MS) }\end{array}$ & $\begin{array}{l}\text { Calcium } \\
\text { (g/kg MS) }\end{array}$ & $\begin{array}{c}\text { Phosphore } \\
\text { (g/kg MS) }\end{array}$ & $\begin{array}{c}\text { Fluor } \\
\text { (mg/kg MS) }\end{array}$ \\
\hline I (TAP50C) & $\begin{array}{l}\text { Jeunes } \\
\text { Taureaux }\end{array}$ & $\begin{array}{l}\text { ND } \\
590\end{array}$ & $\begin{array}{l}\text { ND } \\
235\end{array}$ & $\begin{array}{r}\text { ND } \\
83\end{array}$ & $\begin{array}{r}\text { ND } \\
3840\end{array}$ \\
\hline II (TAP50d) & $\begin{array}{l}\text { Jeunes } \\
\text { Taureaux }\end{array}$ & $\begin{array}{l}613 \\
615\end{array}$ & $\begin{array}{l}235 \\
281\end{array}$ & $\begin{array}{l}105 \\
106\end{array}$ & $\begin{array}{l}655 \\
595\end{array}$ \\
\hline III (THP200C) & $\begin{array}{l}\text { Jeunes } \\
\text { Taureaux }\end{array}$ & $\begin{array}{l}614 \\
630\end{array}$ & $\begin{array}{l}231 \\
296\end{array}$ & $\begin{array}{l}98 \\
94\end{array}$ & $\begin{array}{r}845 \\
1120\end{array}$ \\
\hline IV (Témoin) & $\begin{array}{l}\text { Jeunes } \\
\text { Taureau }\end{array}$ & $\begin{array}{l}606 \\
619\end{array}$ & $\begin{array}{l}231 \\
293\end{array}$ & $\begin{array}{l}103 \\
118\end{array}$ & $\begin{array}{r}465 \\
2190\end{array}$ \\
\hline
\end{tabular}

TAP50 $=$ phosphate de Taiba - dose quotidienne de $50 \mathrm{~g}$ en mode continu TAP50d = phosphate de Taiba - dose quotidienne de $50 \mathrm{~g}$ en mode discontinu THP200c $=$ phosphate de Thiès - dose quotidienne de $200 \mathrm{~g}$ en mode continu ND : non déterminé

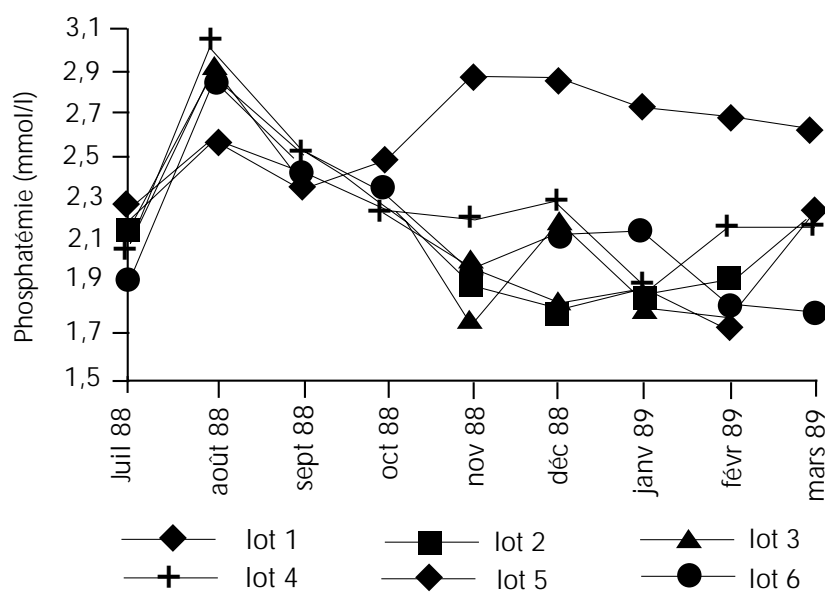

Figure 2 : phosphatémie moyenne des taureaux à Dahra.

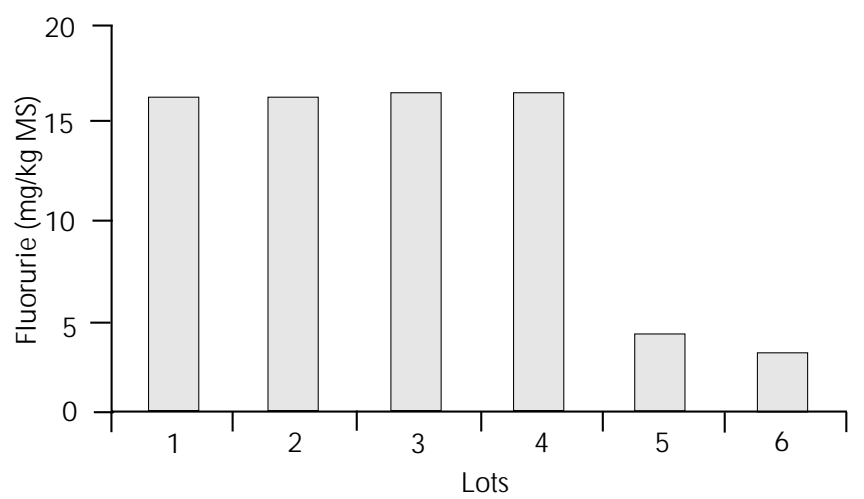

Figure 3 : fluorurie des taureaux à D ahra en avril 1990. 


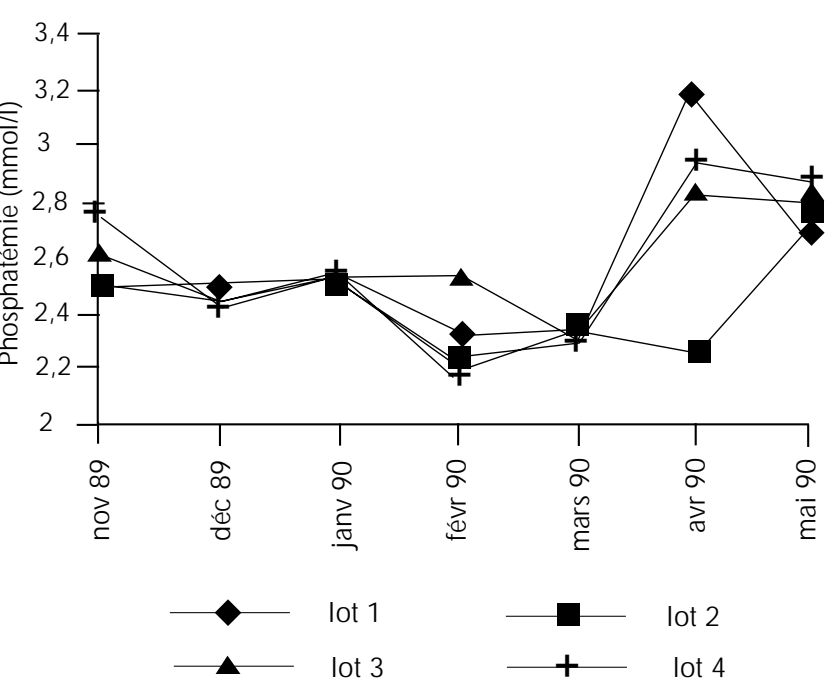

Figure 4 : phosphatémie des veaux à Sangalcam.

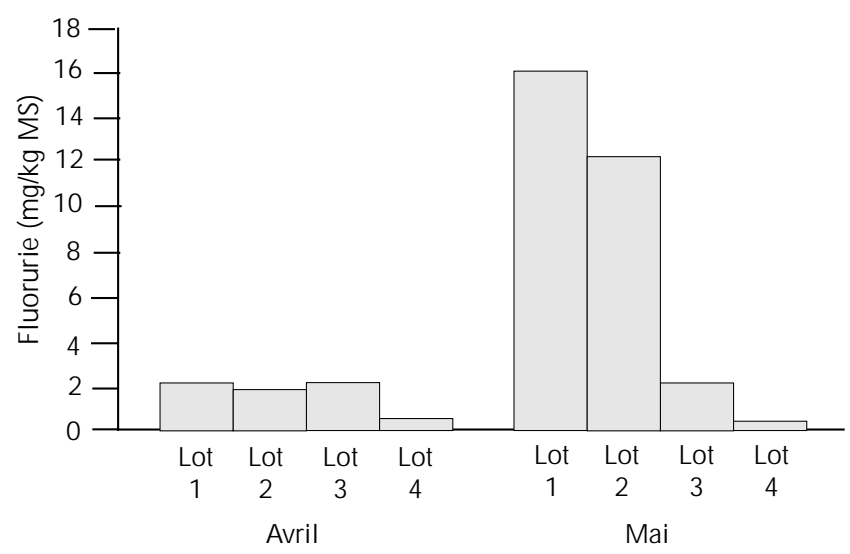

Figure 6 : fluorurie des taureaux à Sangalcam en avril et en mai 1990.

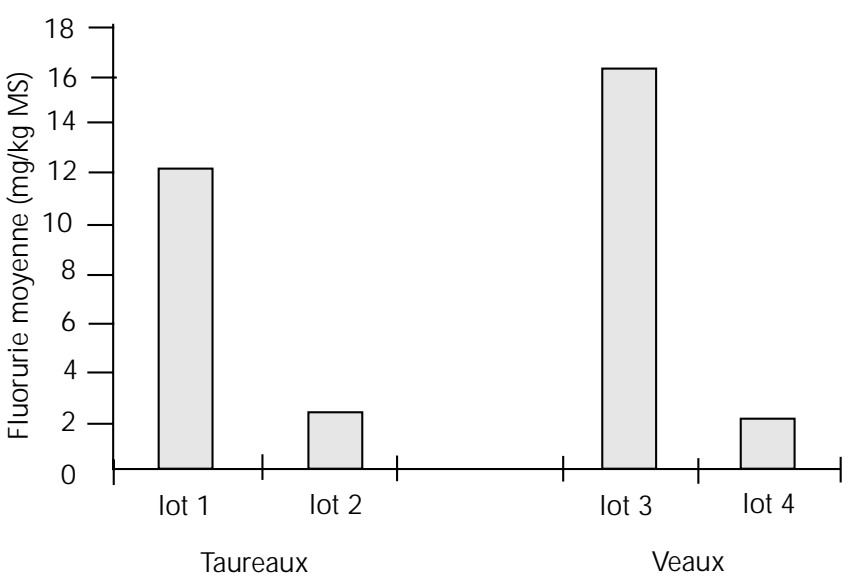

Figure 8 : influence du type de phosphate sur la fluorurie à Sangalcam.

\section{Examen anatomopathologique des os}

Aucune lésion osseuse attribuable à la consommation de phosphates n'a été identifiée par l'examen ante et post mortem de l'appareil osseux des animaux (figures 9 et 10).

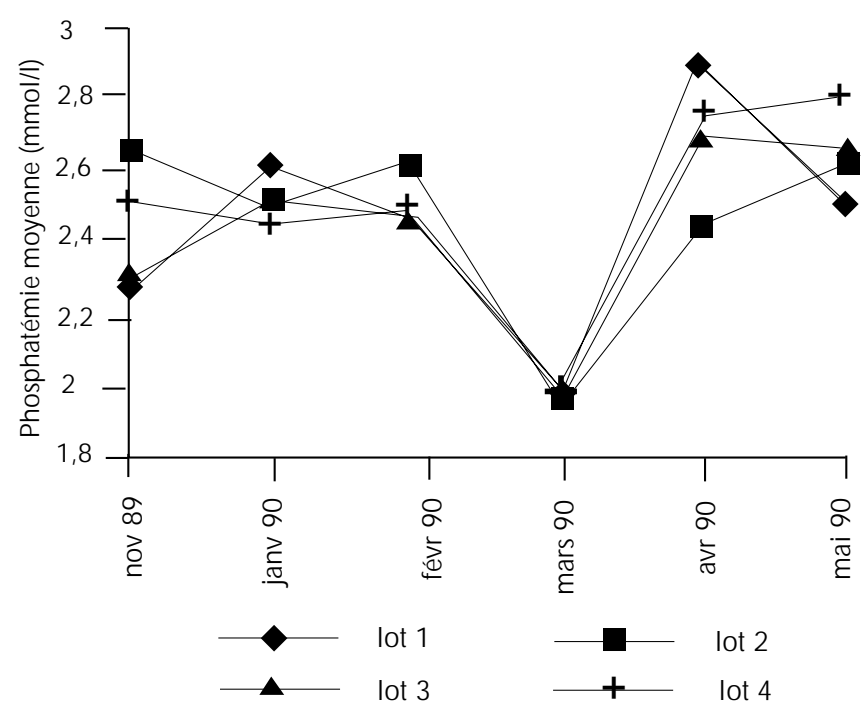

Figure 5 : phosphatémie des taureaux à Sangalcam.

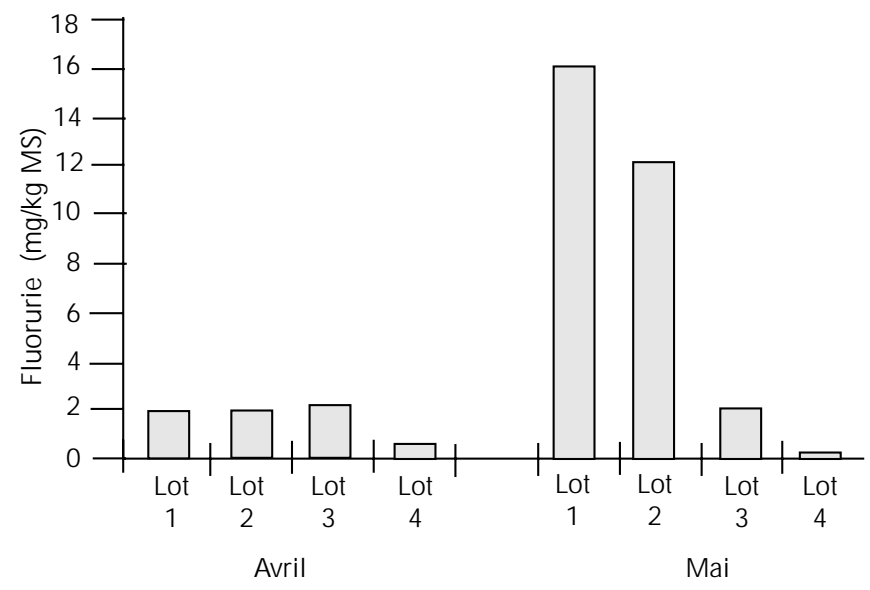

Figure 7 : fluorurie des veaux à Sangalcam en avril et en mai 1990.

\section{Evolution pondérale des animaux}

Tous les lots ont eu une évolution pondérale identique à Dahra (gains moyens quotidiens : $212 \mathrm{~g}$ ) et à Sangalcam (gains moyens quotidiens, $280 \mathrm{~g}$ chez les jeunes et $405 \mathrm{~g}$ chez les taureaux) (figures 11 et 12). Mais la croissance a été sous l'influence d'interactions significatives $\left(\mathrm{P}<0,0001 ; \mathrm{N}=2648\right.$ mesures $\left.; \mathrm{R}^{2}=0,75\right)$ entre le traitement (lot), l'animal, le mois et l'année. Le traitement seul n'a pas eu d'influence significative $(\mathrm{P}<0,05)$ alors que l'année, le mois et l'animal ont significativement $(\mathrm{P}<0,001)$ affecté la croissance des bovins. Les compléments minéraux (phosphates et poudre d'os) n'ont donc pas eu d'influence significative $(\mathrm{P}<0,05)$ sur la croissance pondérale des bovins.

\section{Comportement alimentaire des lots}

A Sangalcam, aucune influence spécifique des compléments minéraux sur le comportement alimentaire des bovins n'a été visuellement constatée, contrairement à Dahra où le pica a uniquement été observé chez les témoins. Le pica étant la cause première du botulisme, cet effet était positif. 

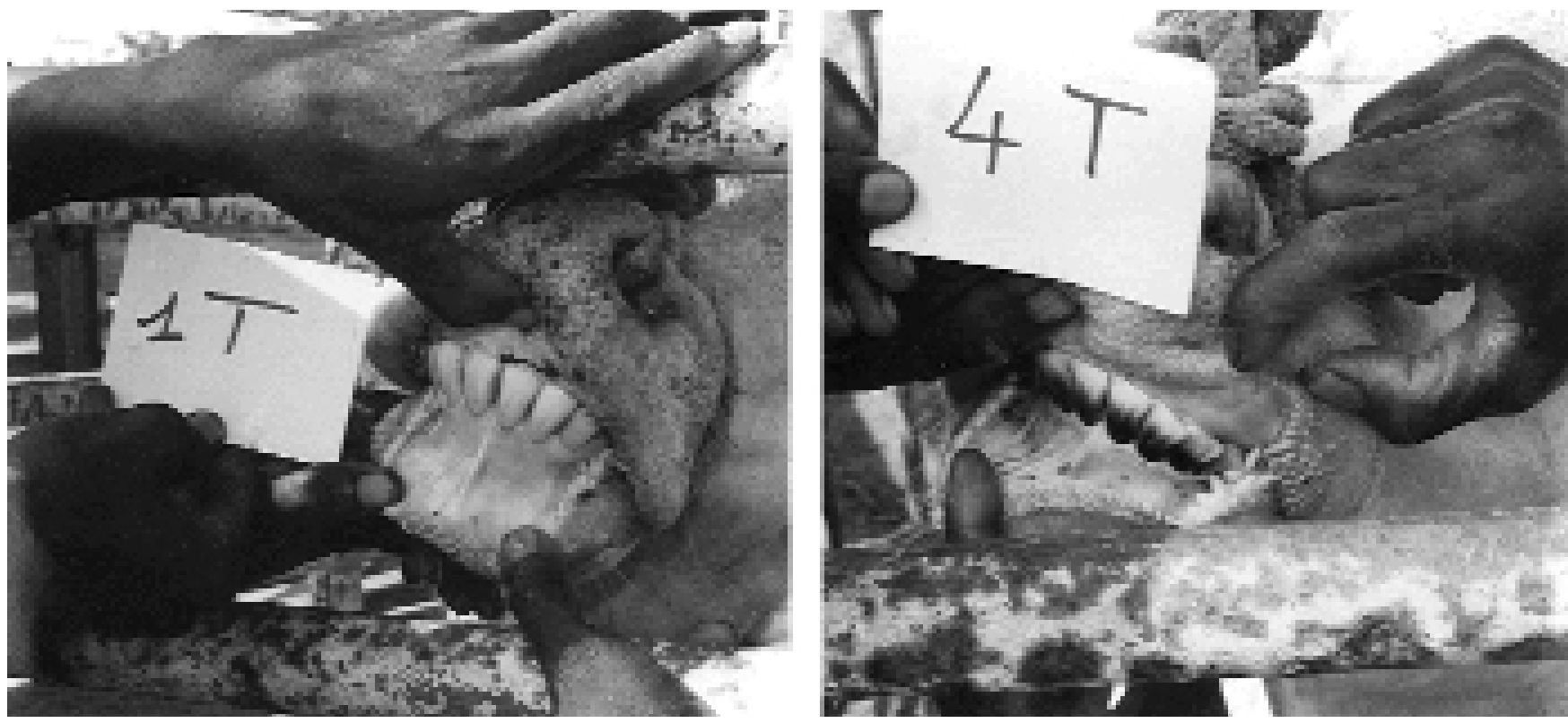

Figure 9 : examen dentaire ; $1 \mathrm{~T}=$ lot 1 (50 g de phosphate de Taiba) ; 4T = lot témoin.
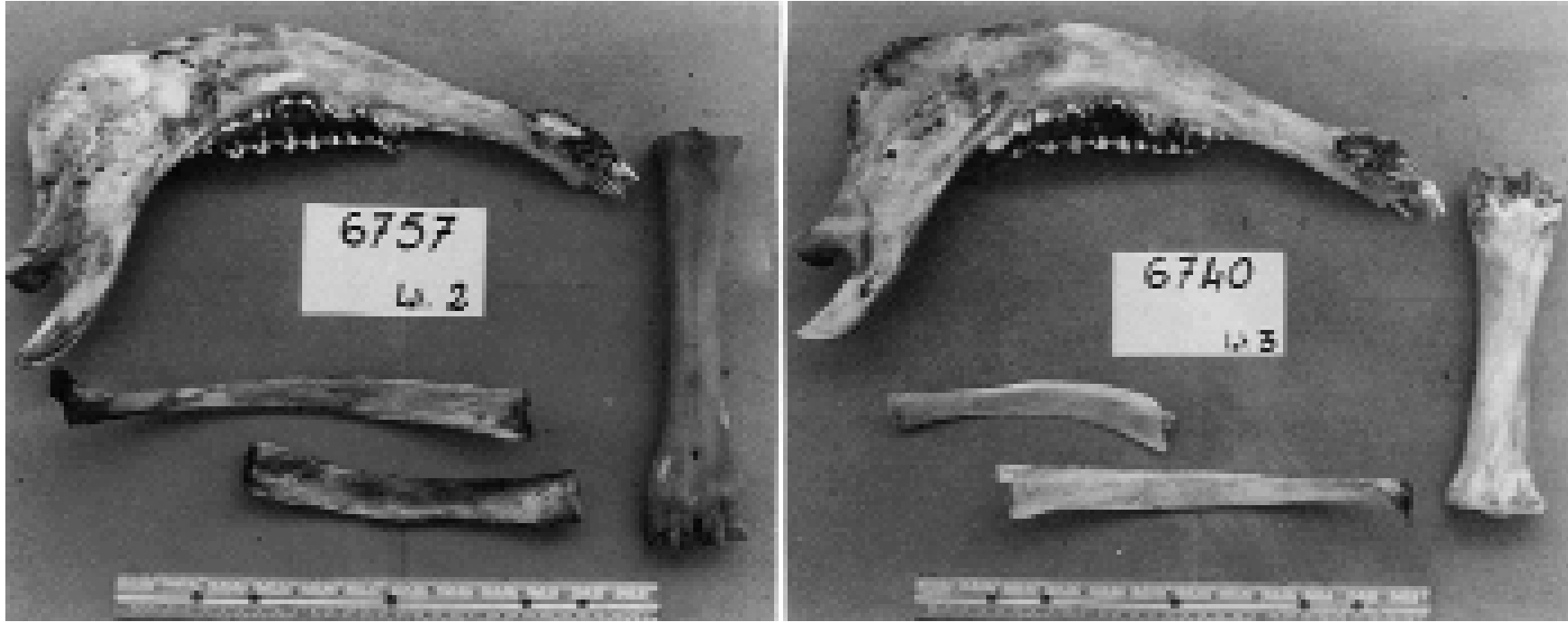

Figure 10 : examen osseux; lot $2=50 \mathrm{~g}$ de phosphate de Taiba ; lot $3=50 \mathrm{~g}$ de phosphate de Thiès.

\section{DISCUSSIO N}

\section{Caractéristiques biochimiques des phosphates naturels et choix du type de gisement pour la supplémentation des ruminants}

Les phosphates naturels testés ont présenté des caractéristiques biochimiques différentes. Cinq critères sont importants pour le choix d'un type de phosphate comme complément minéral : l'appétibilité, la concentration en minéraux, notamment la teneur en calcium par rapport à celle en phosphore, la présence d'autres minéraux favorables ou nocifs et leur disponibilité biologique, le rapport phospho/calcique et la concentration en fluor.

Les phosphates naturels ne sont pas très appétants. Leur pouvoir attractif auprès des animaux ne se manifeste qu'en cas d'état de carence prononcée. Le mélange des compléments à un condiment susceptible d'en améliorer l'appétibilité des animaux est nécessaire. La mélasse mélangée à de la poudre d'os ou aux phosphates a été plus performante que le sel, le son de blé ou la graine de coton. L'incorporation des phosphates à un aliment composé ou complémentaire règle le problème de leur appétibilité. A Sangalcam, ce procédé a permis la consommation totale et régulière des phosphates et de la poudre d'os.

Comparé au phosphate de Thiès (THP), le phosphate de Taiba (TAP) a eu une concentration plus élevée en calcium et en phosphore et une meilleure disponibilité de ces minéraux. Le THP présente un rapport phospho/calcique en faveur du phosphore, ce qui peut être un avantage dans la zone sahélienne fortement carencée en phosphore, ces minéraux ayant toutefois une faible disponibilité biologique. Le TAP était cependant pénalisé par une forte concentration en fluor (3 à 4 p. 100), alors que le THP avait une teneur en fluor peu dangereuse pour l'animal. Il semble recommandable de choisir le TAP dont les risques de toxicité peuvent être modérés par une bonne excrétion urinaire du fluor.

Le déséquilibre phosphocalcique des phosphates peut être réajusté par un mélange avec des composés minéraux plus solubles. Ces mélanges pourraient aussi atténuer les fortes teneurs en fluor et équilibrer la formule du complément minéral en apportant d'autres éléments. 


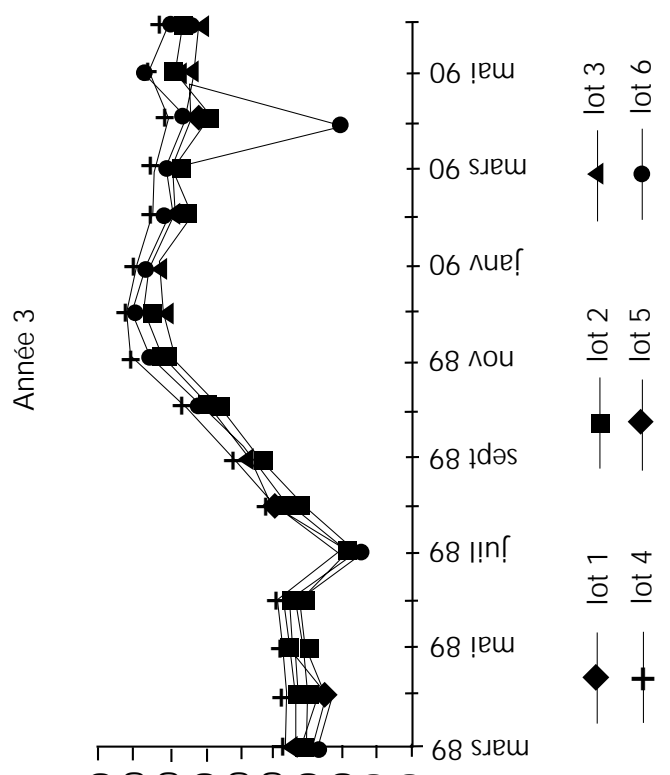

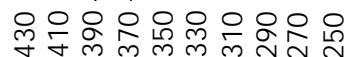

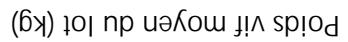

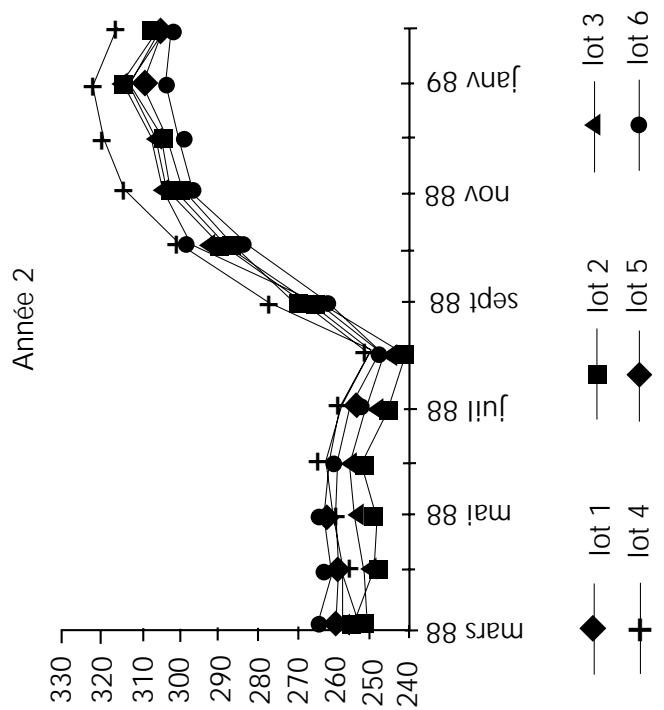

(Бу) ғ이 np uəКou f! sp! sod
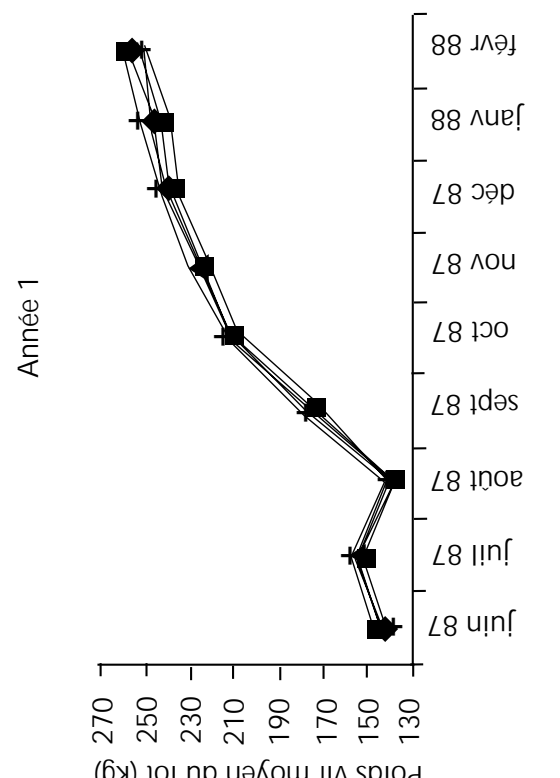

$\begin{array}{ll}m & 0 \\ \stackrel{ }{0} & \underline{0}\end{array}$

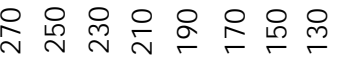

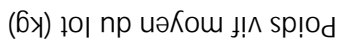

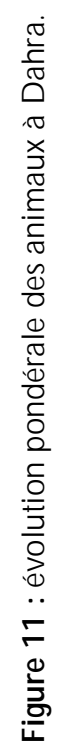

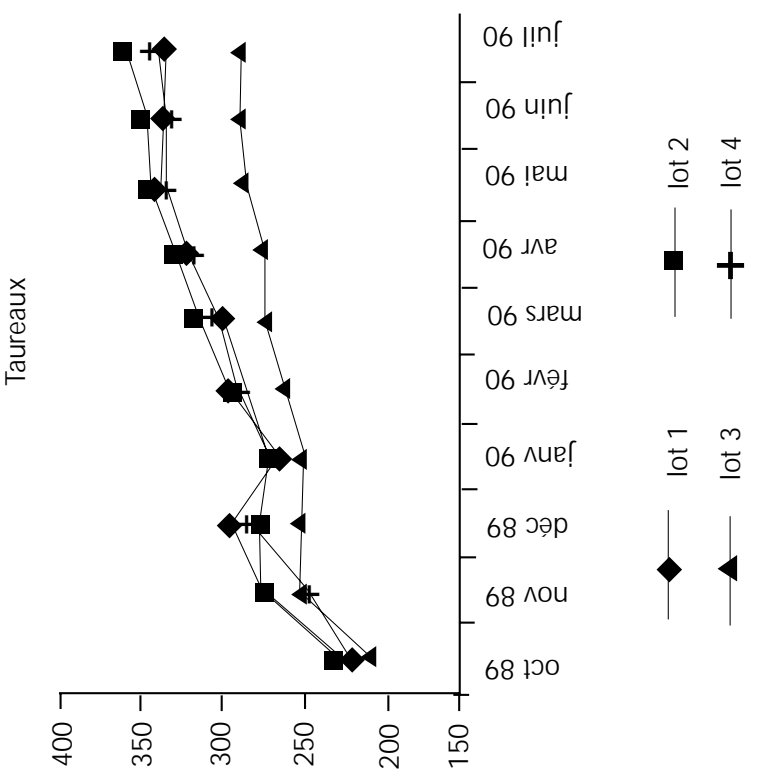

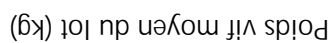

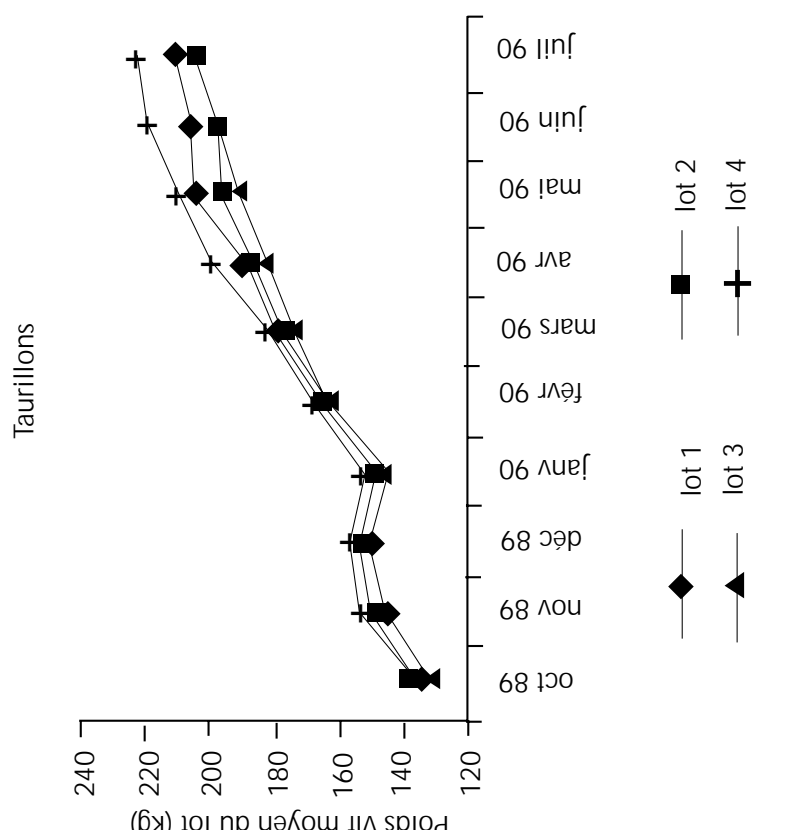

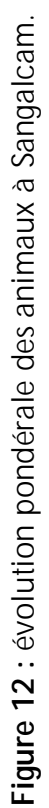

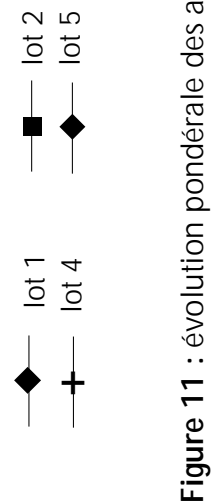


Les phosphates du Sénégal ont des caractéristiques biochimiques différentes de celles des phosphates du Togo dont le fluor semble être plus assimilable si l'on se réfère aux travaux de Serres et Bertaudière (26). Il est donc nécessaire de procéder à l'identification des caractéristiques chimiques des phosphates avant leur utilisation en alimentation du bétail.

\section{Phosphates naturels et santé animale}

L'administration de $50 \mathrm{~g}$ TAP et $100 \mathrm{~g}$ THP en saison sèche pendant trois années successives n'a pas provoqué de maladies graves chez le zébu Gobra. Les lésions osseuses observées correspondaient à des effets légers du fluor (20), mais les phosphates n'en étaient vraisemblablement pas la cause. Cela était peut-être dû au fluor de l'eau ou des aliments qui avaient atteint les seuils critiques et pouvaient, par accumulation, provoquer des lésions subcliniques. Ces lésions n'ont cependant pas entravé la croissance normale de l'animal. L'absence d'une pathologie attribuable à l'effet nocif du fluor traduit une bonne tolérance de ces produits. Les doses de fluor étaient pourtant supérieures au double du maximum admis. Cela est explicable par la présence du fluor sous forme de sels calciques peu solubles contrairement au fluorure de sodium $(10,15,29)$. Si le phosphore des phosphates est d'une utilisation digestive médiocre (15), le fluor semble aussi être peu assimilable. Il est en tout cas très bien déstocké par l'organisme, comme en témoignent les concentrations très élevées des urines en fluor, observées chez les animaux supplémentés. Ainsi, l'élimination urinaire du fluor apparaît comme un mécanisme de protection qui autorise l'ingestion de fortes doses de fluor théoriquement toxiques chez les bovins.

\section{Phosphates naturels et réponse zootechnique}

L'effet non significatif de l'apport de minéraux sur les performances pondérales du zébu Gobra ne traduit pas une mauvaise qualité des phosphates. La poudre d'os, considérée comme un bon complément minéral, n'a pas non plus permis d'améliorer significativement la croissance des bovins. Ces observations confirment également la prévalence d'autres facteurs limitants comme l'énergie, l'azote et d'autres éléments minéraux comme le cuivre et le zinc à prendre en compte dans un programme de supplémentation.

Bien que l'effet bénéfique d'une complémentation minérale sur la productivité des animaux domestiques ait été signalé dans plusieurs pays (7), il ne semble pas y avoir de relation linéaire entre les apports de minéraux et les performances zootechniques. En effet, des irrégularités de la réponse animale ont souvent été rapportées $(2,9,23,24)$. En ce qui concerne la supplémentation en phosphore, les facteurs de variation souvent cités portent sur le site (réponse positive en zone carencée contrairement aux zones autosuffisantes), l'espèce animale (les moutons sont moins sensibles à une carence en phosphore que les bovins), le critère d'appréciation (la baisse du niveau de consommation, les paramètres de la reproduction et la mortalité sont des critères plus sensibles aux carences en phosphore que la croissance pondérale) et le régime alimentaire (la forme chimique présente ainsi que sa digestibilité jouent un rôle déterminant). L'existence de carences associées limite aussi l'influence de l'apport d'un seul élément minéral sur les performances animales. Read et coll. $(23,24)$ ont bien souligné l'importance de l'état nutritionnel des animaux au démarrage de la supplémentation. En effet, des états de carence en phosphore cumulés sur plusieurs années rendent l'animal plus sensible à un apport de phosphore. Si ces apports ne sont insuffisants que pendant une période limitée, la minéralisation osseuse n'est pas encore affectée. Le système de régulation ostéo-hormonal avec l'intervention de la parathormone et de la calcitonine semble adapter l'organisme à cette situation. Enfin la durée de la supplémentation revêt une importance particulière. En Afrique du Sud, Read et coll. $(23,24)$ n'ont enregistré une réponse animale positive qu'au bout de la cinquième année de supplémentation alors qu'à Dahra la supplémentation n'a duré que trois ans.

Dans la station expérimentale de Dahra, le phénomène de carence en phosphore n'était pas marqué. Cela a été confirmé par la croissance du troupeau qui a été modérée malgré un complément minéral reçu facultativement et une très mauvaise année du point de vue de la pluviométrie.

Ces résultats traduisent donc toute la complexité de la réponse animale à la supplémentation minérale, faisant intervenir tant l'effet du milieu (écosystème) que celui de l'animal (espèce, âge, passé nutritionnel, production cible) et de la durée de la supplémentation. La croissance pondérale était moins sensible à la supplémentation minérale que la production laitière ou les paramètres de la reproduction. Ces derniers critères ont fait l'objet d'autres essais à Dahra.

Les phosphates de Taiba et de Thiès aux doses indiquées ici mériteraient donc d'être testés en milieu traditionnel, terrain plus favorable à une démonstration de leur effet positif sur les productions zootechniques. Ils doivent auparavant être équilibrés par l'addition de cuivre et de zinc, éléments dont les déficiences ont été établies dans les zones sahéliennes.

\section{- CON CLU SION}

La consommation quotidienne de $50 \mathrm{~g}$ de phosphate de Taiba et 50 à $200 \mathrm{~g}$ de phosphate de Thiès en période de saison sèche $(9$ mois sur 12 pendant trois ans) a été bien tolérée par les bovins. Ces doses sont sans danger et confirment les résultats de Diallo et coll. (9) et de N'Diaye (22). Elles sont recommandables pour les bovins sur pâturages naturels en distribution continue pendant la saison sèche $(9$ mois sur 12) ou chez des animaux en embouche pendant 3 à 6 mois.

Ainsi, les phosphates de Taiba et de Thiès semblent être moins toxiques pour les bovins que les phosphates du Togo étudiés par Serres et Bertaudière (26).

L'influence aussi bien des phosphates que de la poudre d'os sur les performances pondérales n'a pas été significative. Elle a été limitée par de bonnes années pluviométriques avec de bons pâturages et un état de carences non cumulées chez les bovins. L'innocuité des doses appliquées étant vérifiée, il serait recommandable de les appliquer en milieu traditionnel, plus propice à la démonstration de l'effet positif des phosphates sur les productions.

L'association des phosphates à des sources de cuivre et de zinc, éléments déficients dans les zones sahéliennes, devrait les équilibrer et améliorer leur efficacité nutritionnelle.

\section{Remerciements}

Ce travail a été mené avec l'appui financier de l'Organisation mondiale du phosphate (Imphos). Les phosphates de Taiba et de Thiès ont été gracieusement fournis par la Compagnie sénégalaise des phosphates de Taiba et la Société sénégalaise des phosphates de Thiès. Une partie des analyses chimiques concernant le fluor a été effectuée par le groupe Laboratoires du Bureau des recherches géologiques et minières du Sénégal. N'Diaga M'Baye, Antoine Sarr, Antoine Korea, le regretté Amangoné N'Doye et Momar Coumba Bâ ont apporté leur collaboration technique. D.A. Little, M. M'Bodj, D. Richard et A. Benjelloun ont partagé des critiques constructives sur le déroulement des travaux lors d'une mission d'évaluation en juin 1988. Les auteurs leur expriment leur profonde gratitude. 


\section{BIBLIO GRAPH IE}

1. BIPEA, 1976. Recueil des méthodes d'analyses des Communautés européennes. Paris, France, BIPEA.

2. CALVET H., FRIOT D., GUEYE I.S., 1976. Supplémentation minérales, alimentaires et pertes de poids des zébus sahéliens en saison sèche. Revue Elev. Méd. vét. Pays trop., 29 : 59-66.

3. CALVET H., PICART P., DOUTRE M., CHAMBRON J., 1965 A phosphorose et botulisme au Sénégal. Revue Elev. Méd. vét. Pays trop. $18: 249-282$

4. CARE A.D., 1977. Recent development in the use of phosphate in animal feeding. In: Proc. 1st Int. Congress on Phosphorus Compounds Rabat, M orocco, O ctober 1977, p. 417-420.

5. CHAPMAN H.L., KASTELIC J., ASTON J.C., CATRON D.V., 1955. A comparison of phosphorus from different sources for growing and finishing swine. J. Anim. Sci., 14: 1073-1085.

6. CHURCH D.C. Ed., 1984. Digestive physiology and nutrition of ruminants, Vol 2. Englewood Cliffs, NJ, USA, Prentice-Hall, p. 358-362.

7. CONRAD J.H., MCDOWELL J.R., ELLIS G.L., LOOSLI J.K., 1985 Minéraux pour les ruminants sur pâturages des régions tropicales. Gainesville, Floride, Etats-Unis, Centre pour l'agriculture tropicale Université de Floride, Agence des Etats-U nis pour le Développement international, 96 p. (Bulletin du Departement de zootechnie)

8. DIALLO I., M'BAYE N., GUERIN H., 1983. Effet d'une complémentation minérale et azotée sur la productivité des troupeaux naisseurs de la zone sylvopastorale. Premiers résultats. Rapport technique. Dahra, Sénégal, Isra-Lnerv/Crz, 20 p. (Physiol. Nutr. $n^{\circ}$ 45)

9. DIALLO I., SOW R., NGOMA A., DIOP B., 1985. Utilisation des blocs mélasse urée comportant trois sources de phosphates naturels (Thiès, Taiba, Matam) dans un essai de complémentation destiné à des génisses Gobra en élevage extensif. Dahra, Sénégal, Isra-Crz, p. 83-90. (Rapport annuel)

10. FERRANDO R., 1982. Le phosphore et la vie animale. Académie d'agriculture de France. In : Extrait du procès verbal de l'Académie d'agriculture de France, Paris, séance du 10 février 1982, p. 290-307.

11. FERRANDO R., 1994. Le phosphore dans le règne animal. In : Actes de la $4^{\mathrm{e}}$ conférence internationale de I'Institut mondial du phosphate (Phosphore, vie et environnement), Ghent, Belgique, 8-11 septembre 1994, p. 103-108.

12. FERRANDO R., 1994. Phosphorus deficiency in relation to animal pathology. In : Actes de la 4 e conférence internationale de I'Institut mondial du phosphate (Phosphore, vie et environnement), Ghent Belgique, 8-11 septembre 1994, p. 294-306.

13. FRIOT D., 1969. Rapport sur l'analyse chimique des eaux de forages profonds. Dakar-Hann, Sénégal, Lnerv, 10 p. (Convention 20/C/66/A FAC) IEM VT LNERV.

14. FRIOT D., CALVET H., 1971. Etudes complémentaires sur les carences minérales rencontrées dans les troupeaux du Nord Sénégal.
15. GUEGUEN L., 1961. Valeur comparée des phosphates minéraux comme source de phosphore pour les animaux. Ann. Zootech., 10 : 177-196.

16. GUEGUEN L., 1970. Les critères de qualité nutritionnelle des compléments minéraux en alimentation animale. Bull. Soc. sci. Hyg aliment, 58 : 116-129.

17. GUEGUEN L., 1994. Biodisponibilité et bilan du phosphore chez I'animal. In : Actes de la $4^{e}$ conférence internationale de l'Institut mondial du phosphate (Phosphore, vie et environnement), Ghent, Belgique, 8-11 septembre 1992, p. 276-293.

18. GUERIN H., 1987. Alimentation des ruminants domestiques sur pâturages naturels sahéliens et sahélo-soudaniens : étude méthodologique dans la région du Ferlo au Sénégal. Thèse Doct. Ing. agro., EN SA, M ontpellier, France, $211 p$

19. MAUZAC M., GUERARD F., MATHIEU J., 1976. Dosage du fluor et de l'amoniaque par électrode spécifique. Analysis, 4 : 326-329.

20. MILHAUD G., GODFRAIN J.C., 1975. La fluorose bovine d'origine industrielle. Rec. Méd. vét. Alfort, 191 : 265-272.

21. MONCIARDINI C., 1964 Sédimentation éocène au Sénégal ; le phosphate de chaux de la région de Louga - Linguère. Dakar, Sénégal, Bureau de recherches géologiques et minières, 22 p. (Rapport DAK 64 A4)

22. N'DIAYE V., 1985. Utilisation des phosphates naturels dans I'alimentation des bovins tropicaux. Cas du Sénégal. Thèse Méd. vét. EISM V, Dakar, Sénégal, 85 p. ( $\left.n^{\circ} 21\right)$

23. READ M.V.P., ENGELS E.A.N., SMITH W.A., 1986. Phosphorus in the grazing ruminant. 1 . The effect of supplementary $P$ on sheep at Armoedsvlakte. S. Afr. J. Anim. Sci., 16: 1-6.

24. READ M.V.P., ENGELS E.A.N., SMITH W.A., 1986. Phosphorus and the grazing ruminant 2 . The effect of supplémentary $P$ on cattle at $G$ len and Armoedsvlakte. S. Afr. J. Anim. Sci., 16: 7-12.

25. SAS, 1988. SAS U ser's guide: Statistics, version 5. Cary, NC, USA, SAS Institute Inc.

26. SERRES H., BERTAUTIERE L., 1979. Essais de distributions discontinues de phosphates naturels dans l'alimentation des bovins tropicaux. Revue Elev. Méd. vét. Pays trop., 32 : 391-399.

27. SHUPE J.L., 1963. Diseases of cattle. Santa Barbara, CA, USA, American Veterinary Publications.

28. SOCIETE D'ETUDE ET D'APPLICATION DES MINERAIS DE THIES, 1967. Le Polyfos dans l'alimentation des vaches laitières. Résultats de quatre années de démonstration. Rapport technique. Thiès, Sénégal, Smt, $3 p$

29. UNDERW OOD E.J., 1956. Trace element in human and anima nutrition. N ew York, NY, USA, Academic Press Publishers, p. 312-340.

Reçu le 18.8.98, accepté le 27.8.99 


\section{Summary}

Fall S.T., Sawadogo G., Diop M. Rock phosphates and cattle feeding in Sahel. I. Influence on the Gobra zebu health and growth

Two trials were carried out in Senegal to specify the utilization mode of rock phosphates in animal feeding. Gobra zebus were supplemented with phosphates from Taiba (TAP 3 to $4 \%$ of fluorine dry matter) and Thies (THP 0.8 to $1 \%$ fluorine DM), in Dahra and in a controlled grazing environment in Sangalcam, in the Sahel area of Senegal. In trial 1 Gobra zebus were supplemented for three years on natural pastures at Dahra CRZ. Groups 1 and 2 were supplemented continuously and on alternate months, respectively, with $50 \mathrm{~g}$ TAP (group 2 low phosphate intake resulted in the alternate mode being suspended in the course of year 2). Groups 3 and 4 were continuously supplemented with 50 and $100 \mathrm{~g}$ THP, respectively, and group 5 continuously with $65 \mathrm{~g}$ bone meal. Group 6 was not supplemented as a control. The animals were supplemented during the dry season eight to nine months per year (between 0 ctober and June). In trial 2 four groups were supplemented in a controlled grazing environment during nine months. Groups 1, 2 and 3 were continuously supplemented with $50 \mathrm{~g}$ TAP, alternately with $50 \mathrm{~g} \mathrm{TAP}$, and continuously with $200 \mathrm{~g}$ THP, respectively. Group 4 was not supplemented as a control. In both trials the authors monitored cattle phosphate consumption (daily), performed double weighing (monthly for each group), and carried out clinical examinations on their general state of health, locomotion apparatus and teeth to detect signs of fluorosis. Biochemical analyses were performed to evaluate phosphorus in blood and fluorine concentrations. Results showed a good tolerance of the used doses. The influence of mineral supplementation on animal growth was not however significant $(P<0.05)$. As a result of its innocuousness, to demonstrate the beneficial effect of mineral supplementation on Gobra zebu zootechnical performances a longer trial period on traditional farm herds seems appropriate.

Key words: Cattle - Gobra Zebu - Rock phosphate - Food enrichment - Pasture - Sahel - Senegal.

\section{Resumen}

Fall S.T., Sawadogo G., Diop M. Fosfatos naturales y alimentación del ganado. I. Influencia sobre la salud y el crecimiento del cebú Gobra

Se llevaron a cabo dos estudios en Senegal, con el fin de precisar el modo de utilización de los fosfatos naturales en la alimentación animal. Se suplementaron cebúes Gobra con el fosfato de Taiba (TAP 3 a $4 \%$ de materia seca de fluor) y el de Thies (THP 0,8 a 1 \% MS de fluor), en Dahra y en un medio controlado en Sangalcam, en la zona sahelina de Senegal. En el ensayo 1, la suplementación de los cebúes Gobra se efectuó en pastoreo natural, en el CRZ de Dahra, durante tres años. Las dosis administradas fueron de $50 \mathrm{~g}$ de fosfatos de Taiba, según los métodos continuo y discontinuo, respectivamente, para los lotes 1 y 2 (para este último lote la distribución según el método discontinuo se abandonó durante el curso del segundo año, debido a una baja ingestión del fosfato). Estas fueron (mediante el método continuo) de $50 \mathrm{y}$ de $100 \mathrm{~g}$ de fosfato de Thies, respectivamente, para los lotes 3 y 4 , y de $65 \mathrm{~g}$ de harina de huesos para el lote 5 . El lote 6 , testigo, no recibió ningún suplemento. Los animales se suplementaron durante la estación seca, durante ocho a nueve meses por año (de octubre a junio). En el ensayo 2, cuatro lotes se suplementaron en medio controlado durante nueve meses. Los bovinos recibieron $50 \mathrm{~g}$ de TAP en forma continua, $50 \mathrm{~g}$ de TAP en forma discontinua y $200 \mathrm{~g}$ de THP en forma continua, para los lotes 1,2 y 3 respectivamente. Estos animales fueron comparados al lote 4, testigo, no suplementado. En los dos ensayos las medidas concernieron el control cotidiano del consumo de fosfatos, el seguimiento ponderal mediante una pesada doble mensual de los lotes y exámenes clínicos del estado general, el aparato locomotor y los dientes, con el fin de detectar los signos de fluorosis. Se realizó un control bioquímico para el análisis de la fosfatemia y del contenido de fluor. Los resultados demostraron una buena tolerancia a las dosis administradas. Sin embargo, la influencia de la suplementación mineral sobre el crecimiento de los animales no fue significativa $(P<0,05)$. La inocuidad del producto sugiere un test de larga duración en medio de producción, más propicio para una demostración del efecto benéfico de la suplementación mineral sobre los rendimientos zootécnicos del cebú Gobra.

Palabras clave: Ganado bovino - Cebú Gobra - Fosfato mineral - Enriquecimiento de los alimentos - Pastizal - Sahel Senegal. 